\title{
Efficacy and safety of avapritinib in advanced systemic mastocytosis: interim analysis of the phase 2 PATHFINDER trial
}

\author{
Jason Gotlib ${ }^{1,23 凶}$, Andreas Reiter ${ }^{2,23}$, Deepti H. Radia ${ }^{3}$, Michael W. Deininger $\left({ }^{4} 4\right.$, Tracy I. George ${ }^{5}{ }^{5}$, \\ Jens Panse ${ }^{6}$, Alessandro M. Vannucchi ${ }^{7}$, Uwe Platzbecker ${ }^{8}$, Iván Alvarez-Twose ${ }^{9}$, Andrzej Mital ${ }^{10}$, \\ Olivier Hermine ${ }^{11}$, Ingunn Dybedal' ${ }^{12}$, Elizabeth O. Hexner ${ }^{13}$, Lisa K. Hicks ${ }^{14}$, Lambert Span ${ }^{15}$, \\ Ruben Mesa ${ }^{16}$, Prithviraj Bose ${ }^{17}$, Kristen M. Pettit ${ }^{18}$, Mark L. Heaney ${ }^{19}$, Stephen T. Oh ${ }^{20}$, \\ Jayita Sen ${ }^{21}$, Hui-Min Lin' ${ }^{21}$, Brenton G. Mar ${ }^{21}$ and Daniel J. DeAngelo ${ }^{22}$
}

\begin{abstract}
Advanced systemic mastocytosis (AdvSM) is a rare, KIT D816V-driven hematologic neoplasm characterized by mast cell infiltration and shortened survival. We report the results of a prespecified interim analysis of an ongoing pivotal single-arm phase 2 trial (no. NCT03580655) of avapritinib, a potent, selective KIT D816V inhibitor administered primarily at a once-daily starting dose of $200 \mathrm{mg}$ in patients with AdvSM $(n=62)$. The primary endpoint was overall response rate $(O R R)$. Secondary endpoints included mean baseline change in AdvSM-Symptom Assessment Form Total Symptom Score and quality of life, time to response, duration of response, progression-free survival, overall survival, changes in measures of disease burden and safety. The primary endpoint was successfully met $\left(P=1.6 \times 10^{-9}\right)$, with an ORR of $75 \%$ (95\% confidence interval $\left.57-89\right)$ in 32 response-evaluable patients with AdvSM who had sufficient follow-up for response assessment, including $19 \%$ with complete remission with full or partial hematologic recovery. Reductions of $\geq 50 \%$ from baseline in serum tryptase ( $93 \%$ ), bone marrow mast cells $(88 \%)$ and $K I T$ D816V variant allele fraction $(60 \%)$ were observed. The most frequent grade $\geq 3$ adverse events were neutropenia (24\%), thrombocytopenia (16\%) and anemia (16\%). Avapritinib demonstrated a high rate of clinical, morphological and molecular responses and was generally well tolerated in patients with AdvSM.
\end{abstract}

S ystemic mastocytosis ( $\mathrm{SM})$ is a rare hematologic neoplasm that is associated with the KIT D816V mutation in $~ 95 \%$ of cases. The KIT D816V mutation drives the increased proliferation and accumulation of neoplastic mast cells, leading to severe, debilitating and often unpredictable symptoms and poor quality of life (QoL) ${ }^{1-3}$. In AdvSM, mast cell infiltration leads to organ damage, referred to as 'C-findings' (that is, cytopenias or liver dysfunction), with limited treatment options and poor survival $^{2,4-6}$. AdvSM is comprised of three subtypes: aggressive SM (ASM), SM with an associated hematologic neoplasm (SM-AHN) and mast cell leukemia (MCL) ${ }^{2}$. In SM-AHN, which represents $60-70 \%$ of cases of $\mathrm{AdvSM}^{7,8}$, patients concurrently have both SM and another World Health Organization-defined hematologic neoplasm, usually myeloid (for example, myelodysplastic syndrome, myeloproliferative neoplasm or an overlap $)^{9}$. The KIT D816V mutation is frequently also present in cells comprising the AHN component ${ }^{9}$. Molecular subtyping of patients with AdvSM often reveals a heterogenous genetic landscape, with high-risk mutations in splicing factors, epigenetic regulators and transcription factors such as SRSF2, ASXL1 and RUNX1, respectively ${ }^{10-12}$. Treatment options are very limited, with only the multikinase inhibitor midostaurin and, more recently, the selective KIT kinase inhibitor avapritinib approved for the treatment of AdvSM.

Avapritinib is a selective KIT and platelet-derived growth factor receptor-alpha (PDGFRA) kinase inhibitor with high potency for the KIT D816V and homologous PDGFRA-mutant proteins ${ }^{1}$. As detailed in the accompanying report ${ }^{13}$, avapritinib was investigated in patients with AdvSM in the phase 1 EXPLORER trial

'Division of Hematology, Stanford Cancer Institute/Stanford University School of Medicine, Stanford, CA, USA. ${ }^{2}$ Department of Hematology and Oncology, University Hospital Mannheim, Heidelberg University, Mannheim, Germany. ${ }^{3}$ Guy's \& St Thomas' NHS Foundation Trust, London, UK. ${ }^{4}$ Versiti Blood Research Institute and Division Hematology and Oncology, Medical College of Wisconsin, Milwaukee, WI, USA. ${ }^{5}$ ARUP Laboratories, University of Utah, Salt Lake City, UT, USA. 'Department of Oncology, Hematology, Hemostaseology and Stem Cell Transplantation, University Hospital RWTH Aachen, Aachen, Germany. ${ }^{7}$ Center for Research and Innovation of Myeloproliferative Neoplasms - CRIMM, Azienda Ospedaliera Universitaria Careggi, University of Florence, Florence, Italy. ${ }^{8}$ Leipzig University, Leipzig, Germany. ${ }^{9}$ Institute of Mastocytosis Studies of Castilla-La Mancha, Spanish Reference Center of Mastocytosis, Toledo, Spain. ${ }^{10}$ Department of Hematology and Transplantology, Medical University of Gdansk, Gdansk, Poland. "Department of Hematology, CEREMAST, Necker-Enfants Malades Hospital, APHP, and Imagine Institute, INSERM U1163, Paris University, Paris, France. ${ }^{12}$ Department of Hematology, Oslo University Hospital, Oslo, Norway. ${ }^{13}$ Abramson Cancer Center, University of Pennsylvania, Philadelphia, PA, USA. ${ }^{14}$ Division of Hematology/Oncology, St. Michael's Hospital, University of Toronto, Toronto, Ontario, Canada. ${ }^{15}$ Department of Hematology, University Medical Center Groningen, University of Groningen, Groningen, the Netherlands. ${ }^{16}$ Mays Cancer Center at UT Health San Antonio MD Anderson, San Antonio, TX, USA ${ }^{17}$ The University of Texas MD Anderson Cancer Center, Houston, TX, USA. ${ }^{18}$ University of Michigan, Ann Arbor, MI, USA. ${ }^{19}$ Columbia University Medical Center, New York, NY, USA. ${ }^{20}$ Siteman Cancer Center at Barnes-Jewish Hospital and Washington University School of Medicine, St. Louis, MO, USA. ${ }^{21}$ Blueprint Medicines Corporation, Cambridge, MA, USA. ${ }^{22}$ Department of Medical Oncology, Dana-Farber Cancer Institute, Boston, MA, USA. ${ }^{23}$ These authors contributed equally: Jason Gotlib, Andreas Reiter. ${ }^{凶}$-mail: jason.gotlib@stanford.edu 
(no. NCT02561988) ${ }^{14}$. In this trial, avapritinib exhibited an ORR of 75\% by modified (m)IWG-MRT-ECNM (International Working Group-Myeloproliferative Neoplasms Research and Treatment and European Competence Network) criteria, including 36\% with complete remission with full (CR) or partial hematologic recovery (CRh), with a median follow-up of 23 months. Responses were seen at all starting doses (30-400 mg once daily (QD)) and deepened over subsequent cycles, but occurred most rapidly at $200 \mathrm{mg}$ QD and higher. Patients experienced profound reductions in objective measures of mast cell burden, including complete molecular remission of KIT D816V, reversion of mast-cell-related organ damage and improvements in symptoms. Review of safety, rapid reduction of disease burden and response rate led to selection of $200 \mathrm{mg}$ as the optimal dose for patients with AdvSM.

Here, we present the results of a prespecified interim analysis from the PATHFINDER trial ${ }^{15}$, an ongoing, international, multicenter, open-label, single-arm, phase 2 registrational trial (no. NCT03580655) of avapritinib $200 \mathrm{mg}$ QD in adult patients with a centrally confirmed diagnosis of AdvSM. Patients were enrolled into cohort 1 (efficacy evaluable) if they had an evaluable mIWG-MRTECNM C-finding (that is, cytopenias, liver function abnormalities, splenomegaly, ascites, pleural effusion) (Supplementary Table 1) at baseline, or MCL irrespective of the presence of C-findings. The primary endpoint of the trial was ORR, including CR/CRh, partial remission (PR) and clinical improvement (CI), as per mIWGMRT-ECNM criteria (Supplementary Table 2), which was tested against $28 \%$, the ORR of midostaurin, as per IWG-MRT-ECNM criteria $^{16}$. A prespecified interim analysis was performed when 32 patients in cohort 1 had sufficient follow-up for response evaluation (interim analysis efficacy population). In addition, cohort 2 included patients without an evaluable $\mathrm{C}$-finding at baseline (that is, evaluable C-findings that resolved with previous therapy or nonevaluable C-findings such as weight loss or large osteolytic lesions) and were therefore not evaluable for response. All enrolled patients (safety population) were included in secondary endpoints, which included changes in patient symptoms, reduction in measures of disease burden and safety.

\section{Results}

Participants. Between 21 November 2018 and 23 June 2020, 62 patients with prospectively centrally adjudicated AdvSM were enrolled (ASM $(n=9)$, SM-AHN $(n=43)$ and MCL $(n=10))$ and received avapritinib primarily at a starting dose of $200 \mathrm{mg}$ QD ( $n=60$; two patients started at $100 \mathrm{mg}$ QD), across cohort $1(n=52)$ and cohort $2(n=10)$ (Extended Data Fig. 1). Avapritinib was administered continuously in 28-day cycles until progression, intolerance, withdrawal by the investigator or patient or death.

The interim analysis was triggered when 32 response-evaluable patients in cohort 1 achieved sufficient follow-up for confirmed evaluation of response. The median age was 68 years (range, 37-85) and $56 \%$ of patients were male (Table 1). By central assessment, $94 \%$ were positive for the KIT D $816 \mathrm{~V}$ mutation and $53 \%$ carried an additional mutation in at least one of the genes SRSF2, ASXL1 or RUNX1 $(S / A / R)$, which is associated with poor survival in $S^{12}$. The majority of patients (72\%) had received previous antineoplastic therapy, including 53\% with midostaurin (Extended Data Fig. 2 ). Baseline median bone marrow mast cell percentage was $50 \%$ (range, 10-95) and median serum tryptase was $293 \mathrm{ng} \mathrm{ml}^{-1}$ (range, 24-1,600). The median KIT D816V variant allele fraction (VAF) in peripheral blood (which rarely has circulating mast cells) was $15 \%$ (range, 0-45). Median spleen volume was $939 \mathrm{ml}$ (range, 150 2,270 ). Baseline characteristics were generally balanced between the safety population and those included versus not included in the interim analysis efficacy population (Extended Data Fig. 3). In the interim analysis efficacy population, the most common baseline eligible mIWG-MRT-ECNM C-findings (Supplementary Table 1)
Table 1 | Baseline characteristics

\begin{tabular}{|c|c|c|}
\hline & $\begin{array}{l}\text { Safety population } \\
(n=62)\end{array}$ & $\begin{array}{l}\text { Interim analysis } \\
\text { efficacy population } \\
(n=32)\end{array}$ \\
\hline $\begin{array}{l}\text { Median age, years } \\
\text { (range) }\end{array}$ & $69(31-88)$ & $68(37-85)$ \\
\hline Male/female, $n(\%)$ & $34(55) / 28(45)$ & $18(56) / 14(44)$ \\
\hline \multicolumn{3}{|c|}{ ECOG performance status, $n(\%)$} \\
\hline $0-1$ & $43(69)$ & $21(66)$ \\
\hline $2-3$ & $19(31)$ & $11(34)$ \\
\hline \multicolumn{3}{|c|}{ AdvSM subtype as per central assessment, $n(\%)$} \\
\hline ASM & $9(15)$ & $2(6)$ \\
\hline $\mathrm{SM}-\mathrm{AHN}$ & $43(69)$ & $26(81)$ \\
\hline $\mathrm{MCL}$ & $10(16)$ & $4(13)$ \\
\hline \multicolumn{3}{|c|}{ KIT D816V mutation status in peripheral blood by central ddPCR, $n$ (\%) } \\
\hline Positive & $59(95)$ & $30(94)$ \\
\hline Negative & $3(5)$ & $2(6)$ \\
\hline $\begin{array}{l}\text { KIT D816V variant } \\
\text { allele fraction in blood, } \\
\text { median percentage } \\
\text { (range) }\end{array}$ & $18(0-47)$ & $15(0-45)$ \\
\hline
\end{tabular}

SRSF2/ASXL1/RUNX1 mutation as per central assay, $n$ (\%)

$\begin{array}{lll}\text { Positive } & 26(42) & 17(53) \\ \text { Negative } & 36(58) & 15(47)\end{array}$

Previous antineoplastic therapy, $n(\%)$

\begin{tabular}{|c|c|c|}
\hline Any & $42(68)$ & $23(72)$ \\
\hline Midostaurin & $34(55)$ & $17(53)$ \\
\hline Cladribine & $8(13)$ & $4(13)$ \\
\hline Imatinib & $5(8)$ & $4(13)$ \\
\hline Interferon & $6(10)$ & $2(6)$ \\
\hline $\begin{array}{l}\text { Bone marrow biopsy } \\
\text { mast cell burden } \\
\text { median percentage } \\
\text { (range) }\end{array}$ & $45(1-95)$ & $50(10-95)$ \\
\hline $\begin{array}{l}\text { Serum tryptase level, } \\
\text { median } \mathrm{ng} \mathrm{ml}^{-1} \text { (range) }\end{array}$ & $283(24-1,600)$ & $293(24-1,600)$ \\
\hline $\begin{array}{l}\text { Spleen volume, median } \\
\mathrm{ml} \text { (range) }\end{array}$ & $748(44-2,601)$ & $939(150-2,270)$ \\
\hline
\end{tabular}

ddPCR, droplet digital PCR

were splenomegaly (44\%), elevated alkaline phosphatase (41\%) and transfusion-independent anemia (41\%) (Extended Data Fig. 4).

Interim analysis of efficacy. Among 32 patients in the interim analysis efficacy population (median follow-up of 10.4 months), the confirmed ORR (CR/CRh/PR/CI; primary endpoint) was $75 \%$ ( $n=24,95 \%$ confidence interval, 57-89, $\left.P=1.6 \times 10^{-9}\right)$, with six patients (19\%) achieving CRh. Ten patients (31\%) achieved PR and eight (25\%) had CI (Table 2). Responses were observed in all AdvSM subtypes, regardless of exposure to previous therapy (Table 2). Additionally, ORR was similar among patients with $(71 \%(95 \%$ confidence interval, 44-90), 12/17) and without (80\% (95\% confidence interval, 52-96), 12/15) baseline $S / A / R$ mutations. Responses were rapid, with a median time to response of 2 months (range, $0.3-12.2$ ). Responses continued to improve over time (Fig. 1), with a median time to CRh of 5.6 months (range, 1.8-6.1). 
Table 2 | Response rates by adjudicated mIWG-MRT-ECNM response criteria

\begin{tabular}{|c|c|c|c|c|c|c|c|c|}
\hline \multirow{2}{*}{$\begin{array}{l}\text { Best confirmed response, } \\
n(\%)\end{array}$} & \multirow{2}{*}{$\begin{array}{l}\text { All AdvSM } \\
(n=32)\end{array}$} & \multicolumn{3}{|c|}{ AdvSM subtype } & \multicolumn{2}{|c|}{ Previous therapy } & \multicolumn{2}{|c|}{ Previous midostauri } \\
\hline & & $\begin{array}{l}\text { ASM } \\
(n=2)\end{array}$ & $\begin{array}{l}\text { SM-AHN } \\
(n=26)\end{array}$ & $\begin{array}{l}\mathrm{MCL} \\
(n=4)\end{array}$ & $\begin{array}{l}\text { Yes } \\
(n=23)\end{array}$ & $\begin{array}{l}\text { No } \\
(n=9)\end{array}$ & $\begin{array}{l}\text { Yes } \\
(n=17)\end{array}$ & $\begin{array}{l}\text { No } \\
(n=15)\end{array}$ \\
\hline $\mathrm{ORR}(\mathrm{CR}+\mathrm{CRh}+\mathrm{PR}+\mathrm{Cl})$ & $24(75)$ & $2(100)$ & $21(81)$ & $1(25)$ & $17(74)$ & $7(78)$ & $14(82)$ & $10(67)$ \\
\hline $95 \%$ Confidence interval & $57-89$ & $16-100$ & $61-93$ & $1-81$ & $52-90$ & $40-97$ & $57-96$ & $38-88$ \\
\hline \multicolumn{9}{|l|}{ Best response } \\
\hline $\mathrm{CR}$ or $\mathrm{CRh}$ & $6(19)$ & $1(50)$ & $5(19)$ & 0 & $3(13)$ & $3(33)$ & $3(18)$ & $3(20)$ \\
\hline$C R$ & 0 & 0 & 0 & 0 & 0 & 0 & 0 & 0 \\
\hline $\mathrm{CRh}$ & $6(19)$ & $1(50)$ & $5(19)$ & 0 & $3(13)$ & $3(33)$ & $3(18)$ & $3(20)$ \\
\hline PR & $10(31)$ & $1(50)$ & $8(31)$ & $1(25)$ & $7(30)$ & $3(33)$ & $5(29)$ & $5(33)$ \\
\hline $\mathrm{Cl}$ & $8(25)$ & 0 & $8(31)$ & 0 & $7(30)$ & $1(11)$ & $6(35)$ & $2(13)$ \\
\hline Stable disease & $4(13)$ & 0 & $2(8)$ & $2(50)$ & $2(9)$ & $2(22)$ & 0 & $4(27)$ \\
\hline Progressive disease & $1(3)$ & 0 & 0 & $1(25)$ & $1(4)$ & 0 & 0 & $1(7)$ \\
\hline Not evaluable & $3(9)^{a}$ & 0 & $3(12)$ & 0 & $3(13)$ & 0 & $3(18)$ & 0 \\
\hline
\end{tabular}

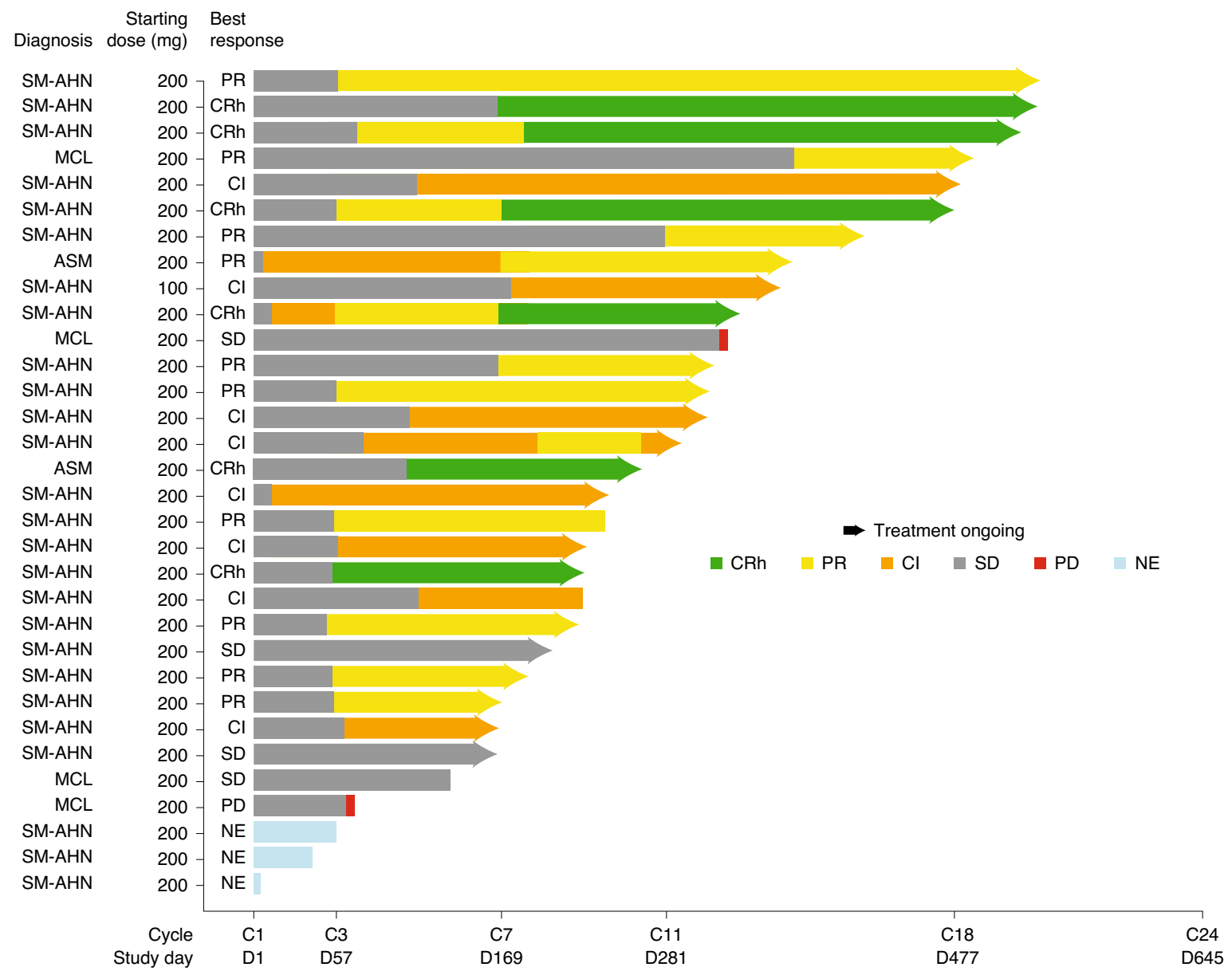

Fig. 1 | Adjudicated mIWG-MRT-ECNM response by cycle. Includes patients in the interim analysis efficacy population ( $n=32)$. SD, stable disease; PD, progressive disease; $N E$, not evaluable.

In the safety population $(n=62)$, consistent and profound reductions in measures of mast cell burden (secondary endpoint) were observed in all enrolled patients with baseline and postbaseline assessments. A reduction of $\geq 50 \%$ in bone marrow mast cells was observed in $88 \%(44 / 50)$ of patients, and $60 \%(30 / 50)$ had elimination of bone marrow mast cell aggregates (Fig. 2a). The serum tryptase level decreased by $\geq 50 \%$ in $93 \%(54 / 58)$ of patients, and $43 \%(25 / 58)$ of patients achieved serum tryptase levels $<20 \mathrm{ng} \mathrm{ml}^{-1}$ (Fig. $2 \mathrm{~b}$ ). 


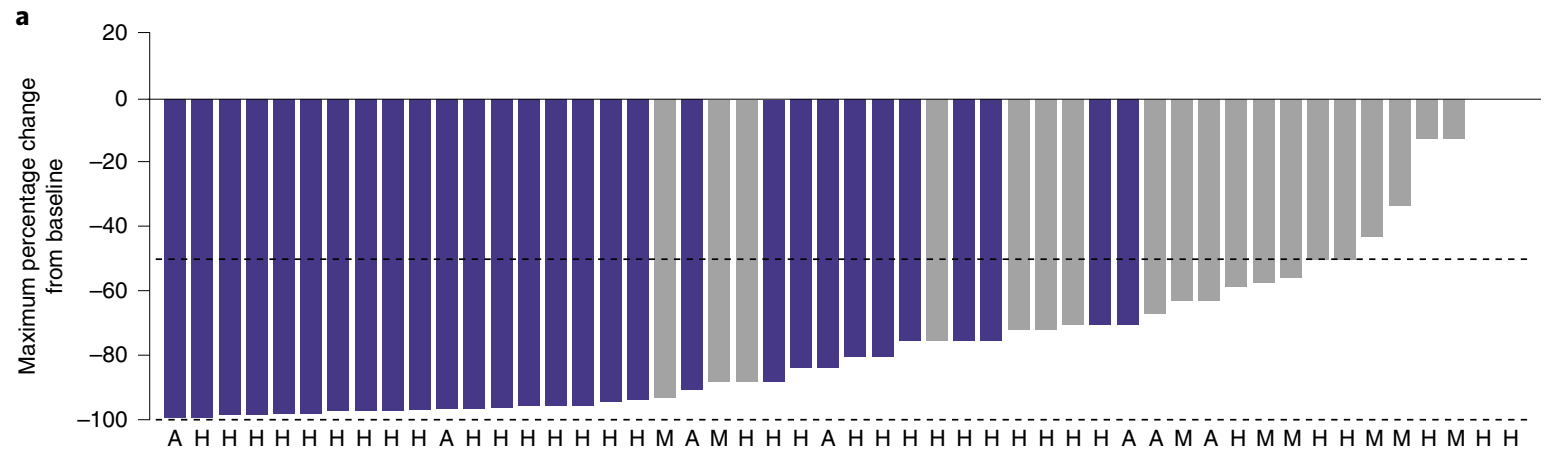

MC aggregates eliminated

A: ASM, H: SM-AHN, M: MCL
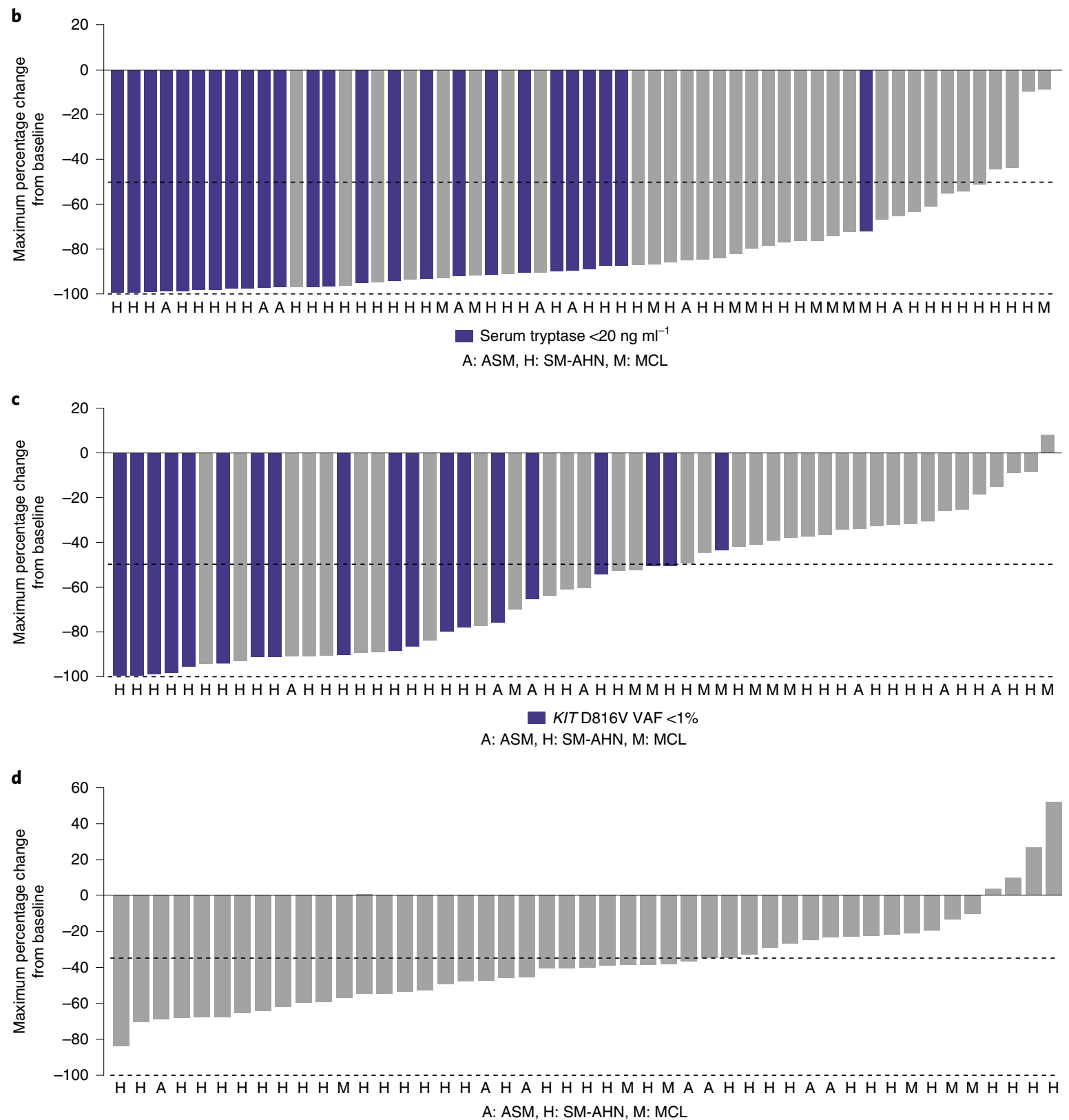

Fig. 2 | Change from baseline in clinicopathological measures of response. a, Bone marrow mast cells. b, Serum tryptase. c, KIT D816V variant allele fraction. d, Spleen volume. MC, mast cell. 
Table 3 | Adverse events (safety population, $n=62$ )

\begin{tabular}{|c|c|c|c|c|}
\hline & & use $A E s$ & & t-related AEs \\
\hline & Any grade & Grade $\geq 3$ & Any grade & Grade $\geq 3$ \\
\hline Any $A E, n(\%)$ & $62(100)$ & $42(68)$ & $57(92)$ & $32(52)$ \\
\hline Peripheral edema & $31(50)$ & $2(3)$ & $26(42)$ & $1(2)$ \\
\hline Periorbital edema ${ }^{b}$ & $30(48)$ & $2(3)$ & $28(45)$ & $2(3)$ \\
\hline Diarrhea & $14(23)$ & $1(2)$ & $7(11)$ & $1(2)$ \\
\hline Vomiting & $11(18)$ & $1(2)$ & $6(10)$ & $1(2)$ \\
\hline Fatigue & $9(15)$ & $2(3)$ & $6(10)$ & $2(3)$ \\
\hline Increased blood alkaline phosphatase & $7(11)$ & $3(5)$ & $2(3)$ & $1(2)$ \\
\hline Hematologic AEs ${ }^{a}, n(\%)$ & & & & \\
\hline Thrombocytopeniab & $28(45)$ & $10(16)$ & $25(40)$ & $9(15)$ \\
\hline AEs of special interest, $n(\%)$ & & & & \\
\hline Cognitive effects & $7(11)$ & 0 & - & - \\
\hline Confusional state & $3(5)$ & 0 & - & - \\
\hline Memory impairment & $3(5)$ & 0 & - & - \\
\hline Cognitive disorder & $2(3)$ & 0 & - & - \\
\hline Intracranial bleeding & $1(2)$ & $1(2)^{d}$ & - & - \\
\hline Subdural hematoma & $1(2)$ & $1(2)^{d}$ & - & - \\
\hline
\end{tabular}

${ }^{a}$ Any-cause AEs in $\geq 15 \%$ (any grade) or $\geq 5 \%$ (grade $\geq 3$ ) of patients are listed. ${ }^{b}$ Pooled terms. 'Grade 4 neutropenia of any cause and treatment-related occurred in 5 ( $8 \%$ ) and 4 ( $6 \%$ ) patients, respectively. ${ }^{\mathrm{d}}$ Grade 4 event that occurred before risk mitigation measures were implemented.

Profound reductions in disease activity beyond mast cells were also observed, with a decrease of $\geq 50 \%$ in absolute monocyte counts in $80 \%(16 / 20)$ of patients with SM and chronic myelomonocytic leukemia (SM-CMML), and a decrease of $\geq 50 \%$ in absolute eosinophil counts in $88 \%(14 / 16)$ of patients with eosinophilia, including all three patients with SM and chronic eosinophilic leukemia (SM-CEL) (Extended Data Fig. 5). Consistent with efficacy against KIT D816V mutant-bearing cells, there was a substantial $(\geq 50 \%)$ reduction in KIT D816V VAF in the peripheral blood in $60 \%(33 / 55)$ of patients; $35 \%(19 / 55)$ of patients achieved a VAF of $<1 \%$ (Fig. 2 c). Spleen volume, which may be greatly enlarged due to involvement by both neoplastic mast cells and $\mathrm{AHN}$, was reduced from baseline by $\geq 35 \%$ in $66 \%$ (31/47) of patients (Fig. 2 d).

The majority of mIWG-MRT-ECNM C-findings in patients in the interim analysis efficacy population resolved from baseline, including $83 \%$ of pleural effusions, $79 \%$ of splenomegaly and $57 \%$ of ascites (Extended Data Fig. 4). Resolution of cytopenia was less frequent, despite elimination of bone marrow mast cell aggregates in the majority of patients, consistent with additional etiologies for cytopenia, such as persistent $\mathrm{AHN}$ and/or avapritinib-related myelosuppression.

Median progression-free survival (PFS) in the interim analysis efficacy population and median overall survival (OS) in the safety population (secondary endpoints) had not been reached at the time of data cutoff. The estimated 6-, 9- and 12-month PFS rates were 91,87 and 79\%, respectively; corresponding OS rates were 94, 86 and $86 \%$.

Safety. As of the data cutoff, $52(84 \%)$ of 62 patients were still on treatment with a median follow-up of 7.0 months (range 5.6-8.1). Reasons for treatment discontinuation included adverse events
(AEs) in six patients (with three considered treatment-related according to the local site investigator (decreased weight, subdural hematoma and bleeding propensity with subcutaneous hematoma)), disease progression as per the investigator in three patients (with one transformation to acute myeloid leukemia and two with worsening AHN) and consent withdrawal in one patient (Extended Data Fig. 1).

The most frequent AEs are presented in Table 3. The most frequent nonhematologic AEs (any grade; grade $\geq 3$ ) were peripheral edema $(50 \% ; 3 \%)$, periorbital edema $(48 \% ; 3 \%)$, diarrhea $(23 \% ; 2 \%)$, nausea $(18 \% ; 2 \%)$ and vomiting $(18 \% ; 2 \%)$. The most frequent hematologic AEs (any grade; grade $\geq 3$ ) were thrombocytopenia ( $45 \%$; $16 \%)$, anemia (32\%; $16 \%)$ and neutropenia $(24 \% ; 24 \%)$, although grade 4 neutropenia (absolute neutrophil count $<0.5 \times 10^{9} \mathrm{l}^{-1}$ ) was uncommon, at $8 \%$. Grade $\geq 3$ treatment-related AEs were reported in $32(52 \%)$ patients, the most frequent of which were neutropenia $(23 \%)$ and thrombocytopenia (15\%). There were three (5\%) deaths due to AEs (disease progression, necrotizing fasciitis and hemorrhagic shock), none of which were considered related to treatment. Cognitive effects (confusional state, memory impairment and cognitive disorder) occurred in seven (11\%) patients (Table 3 ) and were primarily grade $1(n=6)$, with one grade 2 .

There was one (1.6\%) intracranial bleeding (ICB) event (subdural hemorrhage), in a patient with severe thrombocytopenia at baseline (platelets, $49 \times 10^{9} \mathrm{l}^{-1}$ ) who was enrolled before the exclusion (below) of such patients. The patient was treated with avapritinib despite worsening thrombocytopenia (platelets, $33 \times 10^{9} \mathrm{l}^{-1}$ ) and developed a grade 2 subdural hematoma 2 days after a single dose of enoxaparin. The patient was not taking antiplatelet agents and had a relatively normal international normalized ratio and 
a

$\begin{array}{lllllllllllll}\text { a } & n & 56 & 53 & 51 & 49 & 45 & 41 & 36 & 35 & 28 & 24 & 22\end{array}$

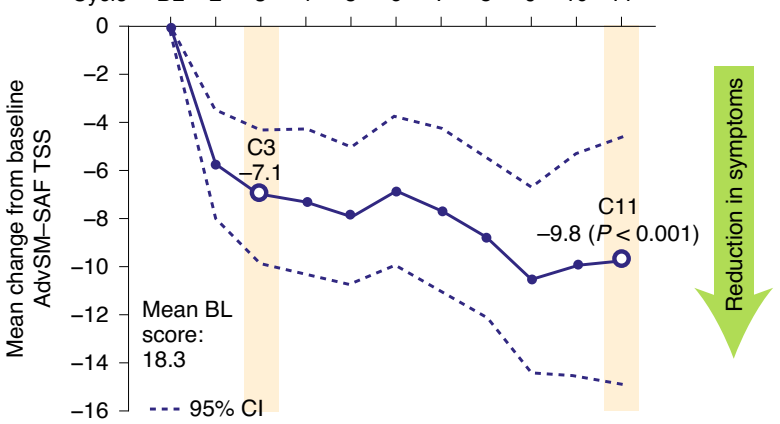

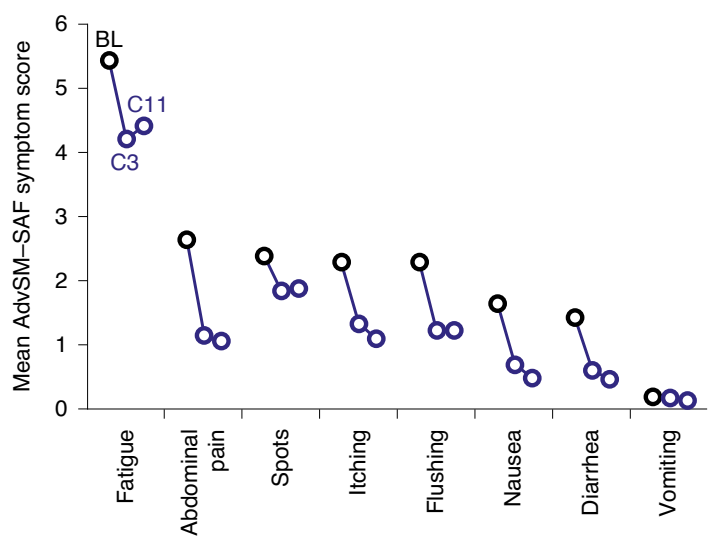

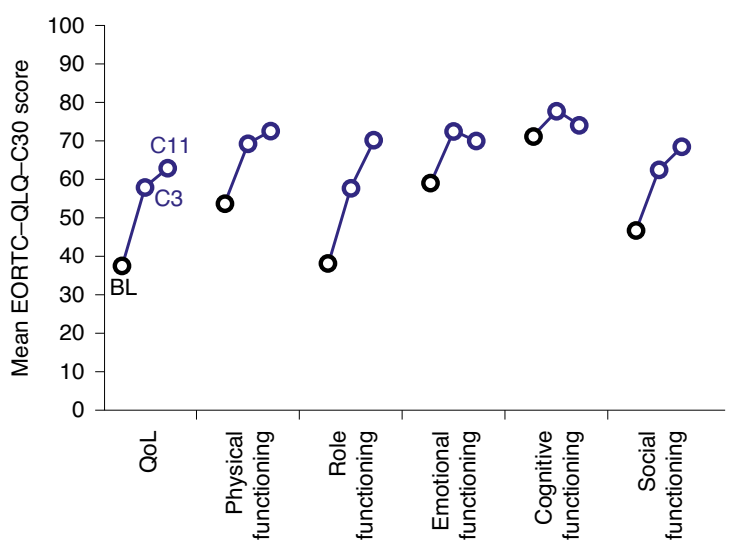

b

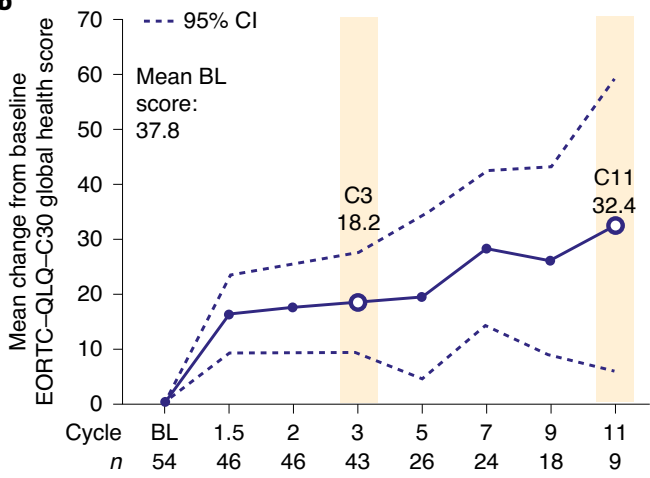

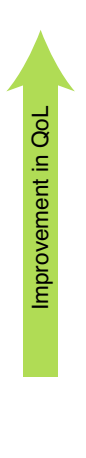

Fig. 3 | Patient-reported outcomes. a, AdvSM-SAF TSS. b, EORTC-QLQ-C30 global health score. BL, baseline. C, cycle.

activated partial thromboplastin time of 1.2 and $28.1 \mathrm{~s}$, respectively, before the ICB event. The patient subsequently recovered from the event and was retreated with avapritinib due to continuing benefit. However, the patient subsequently developed a grade 4 subdural hematoma despite platelets being $167 \times 10^{9} \mathrm{l}^{-1}$, leading to treatment discontinuation.

Safety analysis of this event and similar ICB events in the concurrent phase 1 trial identified patients with baseline severe thrombocytopenia at substantially increased risk of ICB; therefore, patients with platelets $<50 \times 10^{9} \mathrm{l}^{-1}$ at baseline were subsequently excluded from enrollment in both studies. In addition, the studies were amended to include increased platelet count monitoring, updated dose guidance for interruption, support for severely low platelet counts and treatment discontinuation for any grade of ICB events.

In total, six patients (10\%) experienced AEs leading to permanent treatment discontinuation. Two (5\%) patients discontinued due to a treatment-related serious AE (subdural hematoma and bleeding propensity with subcutaneous hematoma). Dose interruptions due to AEs occurred in 34 (55\%) patients, most commonly due to neutropenia (21\%) and thrombocytopenia (16\%) (Extended Data Fig. 6). AEs led to dose reductions in 42 (68\%) patients, most commonly due to neutropenia (19\%) and thrombocytopenia (18\%). The median time to dose reduction was 7.4 weeks, and most patients were taking a daily dose of $100 \mathrm{mg}$ after cycle 3 . The median daily dose was $138 \mathrm{mg}$ (range, 38-240), consistent between patients with or without before exposure to therapy or midostaurin.

Patient-reported outcomes and QoL. Patients' baseline QoL was negatively impacted by their disease, with a pretreatment mean
European Organization for Research and Treatment of Cancer Core QoL Questionnaire C30 (EORTC-QLQ-C30-QoL) score of only 37.8 (range, 0-100, where 0 represents the lowest QoL and 100 the highest) and a high proportion of patients (31\%) with a poor Eastern Cooperative Oncology Group (ECOG) performance status of 2 or 3. Mean and median Patient Global impression of Symptom Severity (PGIS) scores were 2.6 and 3.0, respectively (where 0 represents no symptoms and 4 very severe symptoms). The AdvSM-Symptom Assessment Form (SAF), an AdvSM-specific patient reported outcomes tool, showed that fatigue, abdominal pain and spots were the most severe symptoms, with a mean total symptom score (TSS) of 18.3 , which was the sum of eight possible common symptoms (each scored $0-10$, where 0 represents no symptoms and 10 is the worst imaginable). At baseline, patients had frequent supportive care medication use, including $\mathrm{H} 1$ antihistamines (58\%), H2 antihistamines (39\%) and corticosteroids (32\%). Patients on corticosteroids remained evaluable if their dose did not exceed $20 \mathrm{mg} \mathrm{d}^{-1}$ of prednisone or equivalent.

Patient-reported symptoms, as measured by TSS score, improved rapidly following treatment initiation, dropping by 7.1 points from baseline at cycle $3(n=51)$ and by 9.8 points from baseline at treatment cycle $11(n=22 ; P<0.001)$ (secondary endpoint; Fig. $3 a)$. Mean symptom scores were lower than baseline at cycles 3 and 11 for all SM symptoms, including fatigue, abdominal pain, spots, itching, flushing, nausea, diarrhea and vomiting. Mean and median PGIS scores improved to 1.6 and 2.0 (moderate symptoms that are difficult to ignore), respectively, by cycle 3 and to 1.2 and 1.0 (minimal symptoms that are easy to ignore), respectively, by cycle 11 (secondary endpoint). 
Quality of life, as assessed by EORTC-QLQ-C30, improved on trial with noteworthy improvements in physical (strenuous activity), role (work or household jobs), emotional (irritability, feeling tense and depression), cognitive (memory and concentration) and social (family life and social activities) functioning domains (secondary endpoint; Fig. 3b).

\section{Discussion}

This prespecified interim analysis of the phase 2 PATHFINDER trial demonstrated that avapritinib at a starting dose of $200 \mathrm{mg}$ QD exhibited clinical benefit in patients with AdvSM, confirming findings from the phase 1 EXPLORER trial. In total, $75 \%$ of patients achieved a response regardless of AdvSM subtype, previous therapy or adverse $S / A / R$ comutations, with a rapid median time to response of 2 months. With median follow-up of 10.4 months, $19 \%$ of patients had normalized all baseline evaluable C-findings, eliminated mast cell aggregates and reduced tryptase to $<20 \mathrm{ng} \mathrm{ml}^{-1}$, to achieve CRh. In June 2021, data from this and the phase 1 EXPLORER trial (no. NCT02561988) formed the basis of approval by the United States Food and Drug Administration for the treatment of adults with AdvSM at a recommended starting dose of $200 \mathrm{mg}$ orally QD.

Consistent and profound reductions in mast cell burden (bone marrow mast cells, serum tryptase) and normalization of SM-related organ damage (that is, liver function abnormalities, ascites, spleen size) were observed in patients across all AdvSM subtypes regardless of previous therapy. Although improvements in cytopenias were seen in some patients, including improvement of transfusion-dependent anemia in one patient, cytopenias were less likely to normalize than other C-findings, even in patients who no longer had bone marrow mast cell aggregates. This implies that persistent cytopenias were related to non-mast cell causes, such as drug effect and/or the remaining AHN component.

In addition, avapritinib demonstrated evidence of broader disease activity in patients with SM-AHN, which comprises the majority of AdvSM variants. For example, substantial reductions of monocytosis in $80 \%$ of patients with SM-CMML and eosinophilia in $88 \%$ patients with baseline eosinophilia or SM-CEL were observed. Overall, $60 \%$ of patients had substantial reductions in peripheral blood KIT D816V VAF, consistent with broad activity against both KIT D816V-positive mast cells (which rarely circulate in the blood) and cells derived from the AHN which often harbor KIT D816V, reflecting multilineage involvement of the mutation. Some patients with SM-AHN may need AHN therapy in combination or in sequence with a KIT-inhibitor-based regimen to address the genetic and biologic heterogeneity which underpins this AdvSM subtype. However, most patients had improvement in their AHN with avapritinib alone. This activity supports exploration of avapritinib in other KIT D816V-positive hematologic malignancies.

Patients had marked impairments in QoL at baseline due to their AdvSM symptoms yet reported rapid, durable and substantial reductions from baseline in every AdvSM symptom assessed, and improvements in QoL and functional impairment. Median global symptom severity as measured by PGIS had improved from severe to minimal by cycle 11 .

Avapritinib was generally well tolerated, with few discontinuations due to AEs. Overall, the safety profile at the starting dose of $200 \mathrm{mg}$ QD in the PATHFINDER trial included mostly low-grade fluid retention and gastrointestinal symptoms as the most common nonhematologic AEs. Cytopenias were the most common grade $\geq 3$ AEs and reason for dose reduction, most commonly in patients with baseline cytopenias, and $100 \mathrm{mg}$ was the median daily dose after cycle 3. Cognitive effects, which are dose-related, were relatively uncommon at a starting dose of $200 \mathrm{mg}$.

The incidence of ICB was low (1.6\%), and similar to that observed with avapritinib in patients with GIST ${ }^{17,18}$, probably due to mitigation steps for severe thrombocytopenia which were implemented early in enrollment. These included exclusion of patients with platelets $<50 \times 10^{9} \mathrm{l}^{-1}$, closer monitoring of platelet counts, stricter dose modification guidelines and use of platelet transfusions and growth factors to maintain platelet count to $\geq 50 \times 10^{9} \mathrm{l}^{-1}$. Dose-related, low-grade cognitive events were observed but did not result in substantial dose reductions. The optimal starting dose of $200 \mathrm{mg}$ QD was confirmed in the current trial, because preliminary data generated in the EXPLORER trial suggested that this dose maintained efficacy while improving tolerability.

These data reinforce KIT D816V as a clinically validated drug target in AdvSM. While the multikinase inhibitor midostaurin is already approved for use in patients with AdvSM, there remains a substantial need for improvement in the overall rate, durability and quality of responses ${ }^{19-22}$. Treatment with midostaurin for AdvSM can lead to challenging gastrointestinal side effects, limiting its efficacy. With avapritinib, a potent, selective inhibitor of KIT D816V, the clinical aim in AdvSM is to achieve deeper and enduring clinical, morphological and molecular responses, which could translate into extended survival with improved QoL.

A potential limitation of this prespecified interim analysis is the short duration of follow-up (10.4 months) in a subset of trial patients, which will be addressed in long-term follow-up analyses of the study. It is anticipated that findings from long-term analyses will be consistent with those observed in the phase 1 trial $^{13}$.

In conclusion, interim results from the current phase 2 PATHFINDER trial corroborate the promising and durable outcomes observed in the phase 1 EXPLORER trial. Avapritinib administered at a starting dose of $200 \mathrm{mg}$ QD was well tolerated and led to profound reductions in disease burden, improved patient symptoms and QoL, and elicited deep molecular responses of KIT D816V, highlighting the potential for modification of AdvSM disease natural history.

\section{Online content}

Any methods, additional references, Nature Research reporting summaries, source data, extended data, supplementary information, acknowledgements, peer review information; details of author contributions and competing interests; and statements of data and code availability are available at https://doi.org/10.1038/ s41591-021-01539-8.

Received: 2 June 2021; Accepted: 13 September 2021;

Published online: 6 December 2021

\section{References}

1. Evans, E. K. et al. A precision therapy against cancers driven by KIT/ PDGFRA mutations. Sci. Transl. Med. 9, eaao1690 (2017).

2. Pardanani, A. Systemic mastocytosis in adults: 2019 update on diagnosis, risk stratification and management. Am. J. Hematol. 94, 363-377 (2019).

3. Orfao, A., Garcia-Montero, A. C., Sanchez, L. \& Escribano, L. Recent advances in the understanding of mastocytosis: the role of KIT mutations. Br. J. Haematol. 138, 12-30 (2007).

4. Cohen, S. S. et al. Epidemiology of systemic mastocytosis in Denmark. Br. J. Haematol. 166, 521-528 (2014).

5. Lim, K.-H. et al. Systemic mastocytosis in 342 consecutive adults: survival studies and prognostic factors. Blood 113, 5727-5736 (2009).

6. Valent, P., Akin, C. \& Metcalfe, D. D. Mastocytosis: 2016 updated WHO classification and novel emerging treatment concepts. Blood 129, 1420-1427 (2017)

7. Shomali, W. \& Gotlib, J. Response criteria in advanced systemic mastocytosis: evolution in the era of KIT inhibitors. Int. J. Mol. Sci. 22, 2983 (2021).

8. Jawhar, M. et al. The clinical and molecular diversity of mast cell leukemia with or without associated hematologic neoplasm. Haematologica 102, 1035-1043 (2017).

9. Reiter, A., George, T. I. \& Gotlib, J. New developments in diagnosis, prognostication, and treatment of advanced systemic mastocytosis. Blood 135, 1365-1376 (2020).

10. Awada, H., Thapa, B. \& Visconte, V. The genomics of myelodysplastic syndromes: origins of disease evolution, biological pathways, and prognostic implications. Cells 9, 2512 (2020). 
11. Ustun, C. et al. Advanced systemic mastocytosis: from molecular and genetic progress to clinical practice. Haematologica 101, 1133-1143 (2016).

12. Jawhar, M. et al. Additional mutations in SRSF2, ASXL1 and/or RUNX1 identify a high-risk group of patients with KIT D816V( $\left(^{+}\right.$) advanced systemic mastocytosis. Leukemia 30, 136-143 (2016).

13. DeAngelo, D. J. et al. Safety and efficacy of avapritinib in advanced systemic mastocytosis: the phase 1 EXPLORER trial. Nat. Med. (in the press).

14. (EXPLORER) Study of BLU-285 in Patients with Advanced Systemic Mastocytosis (AdvSM) and Relapsed or Refractory Myeloid Malignancies (2021); https://clinicaltrials.gov/ct2/show/NCT02561988

15. (PATHFINDER) Study to Evaluate Efficacy and Safety of Avapritinib (BLU-285), a Selective KIT Mutation-Targeted Tyrosine Kinase Inhibitor, in Patients with Advanced Systemic Mastocytosis (2020); https://clinicaltrials.gov/ ct2/show/NCT03580655

16. Blueprint Medicines (Netherlands) B.V. AYVAKYT ${ }^{\circledast}$ (avapritinib). Summary of product characteristics. https://ir.blueprintmedicines.com/news-releases/ news-release-details/blueprint-medicines-announces-european-commissionapproval (2020).

17. Heinrich, M. C. et al. Avapritinib in advanced PDGFRA D842V-mutant gastrointestinal stromal tumour (NAVIGATOR): a multicentre, open-label, phase 1 trial. Lancet Oncol. 21, 935-946 (2020).

18. Kang, Y. K. et al. Avapritinib versus regorafenib in locally advanced unresectable or metastatic GI stromal tumor: a randomized, open-label phase III study. J. Clin. Oncol. https://ascopubs.org/doi/full/10.1200/JCO.21.00217 (2021).
19. Novartis Europharm Limited. RYDAPT (midostaurin). Summary of product characteristics. https://www.ema.europa.eu/en/medicines/human/EPAR/ rydapt (2021).

20. Novartis Pharmaceuticals Corporation. RYDAPT (midostaurin). Prescribing information. https://www.hcp.novartis.com/products/rydapt/ acute-myeloid-leukemia/dosing-administration/ (2021).

21. Gotlib, J. et al. Efficacy and safety of midostaurin in advanced systemic mastocytosis. N. Engl. J. Med. 374, 2530-2541 (2016).

22. Stone, R. M. et al. Midostaurin plus chemotherapy for acute myeloid leukemia with a FLT3 mutation. N. Engl. J. Med. 377, 454-464 (2017).

Publisher's note Springer Nature remains neutral with regard to jurisdictional claims in published maps and institutional affiliations.

(c) (i)

Open Access This article is licensed under a Creative Commons

Attribution 4.0 International License, which permits use, sharing, adaptation, distribution and reproduction in any medium or format, as long as you give appropriate credit to the original author(s) and the source, provide a link to the Creative Commons license, and indicate if changes were made. The images or other third party material in this article are included in the article's Creative Commons license, unless indicated otherwise in a credit line to the material. If material is not included in the article's Creative Commons license and your intended use is not permitted by statutory regulation or exceeds the permitted use, you will need to obtain permission directly from the copyright holder. To view a copy of this license, visit http://creativecommons. org/licenses/by/4.0/.

(C) The Author(s) 2021 


\section{Methods}

Participants. Eligible patients were $\geq 18$ years of age and with a diagnosis of AdvSM confirmed by central review. The primary efficacy endpoint of ORR as per mIWG-MRT-ECNM criteria $^{23}$ required an evaluable baseline C-finding (Supplementary Table 1), and these patients were enrolled into cohort 1. Patients who did not have an evaluable C-finding at baseline could not be assessed for the primary endpoint and were enrolled into cohort 2 , with the exception of patients with MCL who, as per criteria, were enrolled in cohort 1 and evaluated for reductions in disease burden. As a result of emerging evidence that an increased risk of ICB is associated with grade 3 thrombocytopenia, the protocol was amended to exclude patients with baseline platelet count $<50 \times 10^{9} \mathrm{l}^{-1}$.

Additional inclusion criteria were as follows: patients with SM-AHN who had received previous treatment for the AHN component of disease if, in the opinion of the investigator, such therapy was appropriate; bone marrow biopsy taken within 56 days of cycle 1 , day 1 (C1D1); serum tryptase levels $\geq 20 \mathrm{ng} \mathrm{ml}^{-1}$; ECOG performance status $0-3$; discontinued cytoreductive therapy due to disease progression, refractory disease, lack of efficacy or intolerance if receiving therapy within the preceding 12 weeks; stable dose of nonantineoplastic SM therapies or corticosteroids ( $\leq 20 \mathrm{mg} \mathrm{d}^{-1}$ prednisone or equivalent) for $\geq 14$ days before C1D8 and able to provide written informed consent. Patients were excluded for the following reasons: received previous treatment with avapritinib; received any cytoreductive therapy or an investigational agent $<14$ days and, for cladribine, interferon alpha, pegylated interferon and any antibody therapy, $<28$ days before obtaining screening bone marrow biopsy; received previous radiotherapy or any hematopoietic growth factor within 14 days before screening bone marrow biopsy; requires concomitant medication that is a strong inhibitor, strong inducer or moderate inducer of cytochrome P450 3A4; had a major surgical procedure within 14 days of the first dose of study drug; is a candidate for allogeneic hematopoietic stem cell transplantation for treatment of SM; eosinophilia and known positivity for the FIP1L1-PGDFRA fusion, unless the patient has demonstrated relapse or progressive disease on previous imatinib therapy; has history of another primary malignancy (within 3 years before the first dose of study drug), cerebrovascular accident or transient ischemic attacks (within 1 year before the first dose of study drug), or seizure disorder; has abnormal laboratory findings or QT interval corrected using Fridericia’s formula $>480 \mathrm{~ms}$; has known risk or recent history of ICB; has a primary brain malignancy or metastases to the brain; has clinically significant, uncontrolled cardiovascular disease; unless postmenopausal (females) or surgically sterile; is unwilling to abstain from sexual intercourse or employ highly effective contraception from the first dose of study drug and for at least 6 weeks after the last dose of study drug; pregnant or breastfeeding; hypersensitivity to avapritinib or to any of the excipients; unwilling or unable to comply with the study procedures or requirements; and participation in another interventional study.

Trial design and treatment. PATHFINDER (ClinicalTrials.gov identifier no. NCT03580655) is an ongoing, international, multicenter, open-label, single-arm, phase 2 registrational trial conducted in North America and Europe. Avapritinib was administered at a starting dose of $200 \mathrm{mg}$ QD in 28-day cycles until progression, intolerance, withdrawal by the investigator or patient or death. Dose modification to as low as $25 \mathrm{mg}$ QD was allowed as per prespecified criteria. Dose increases to $300 \mathrm{mg}$ QD were allowed for lack of efficacy and dose interruptions for platelet counts $<50 \times 10^{9} \mathrm{l}^{-1}$ were required, although platelet transfusion and growth factor support were allowed on trial.

Previous cytoreductive therapy or investigational agents were permitted if received up to 14 days before the screening bone marrow sample or up to 28 days (cladribine, interferon alpha, pegylated interferon or any antibody therapy) before the screening marrow biopsy. Medications, including palliative and supportive care for disease-related symptoms, were permitted during the study and may include the following classes of agents: histamine receptor $\mathrm{H} 1$ and $\mathrm{H} 2$ blockers; proton pump inhibitors; osteoclast inhibitors (that is, bisphosphonates); leukotriene receptor antagonists; corticosteroids (not exceeding $20 \mathrm{mg} \mathrm{d}^{-1}$ of prednisone or equivalent, and dose must be stable for $\geq 14$ days before C1D8); cromolyn sodium and other mast cell stabilizers; and omalizumab.

Trial outcomes and assessments. The primary endpoint was optimal ORR as per mIWG-MRT-ECNM criteria (Supplementary Table 2). Modifications to the IWGMRT-ECNM criteria were previously described ${ }^{7,13}$. Responses as per mIWGMRT-ECNM criteria were adjudicated by the Study Steering Committee based on data from every trial visit, and required confirmation of $\geq 12$ weeks. Bone marrow samples were assessed by central pathology, and serum tryptase and KIT D816V VAF in the blood were analyzed by central laboratory.

The primary analysis was originally planned after 63 patients in cohort 1 had received at least ten cycles of therapy but, due to the high efficacy observed in the EXPLORER trial, an interim analysis was introduced early in the PATHFINDER trial, to be triggered when 32 patients in cohort 1 (interim analysis efficacy population) had sufficient follow-up to be adjudicated for response as per mIWGMRT-ECNM criteria.

The key secondary endpoint was mean change from baseline in patient-reported TSS of the AdvSM-SAF, a validated measure to assess treatment benefit in AdvSM patients ${ }^{24,25}$. Other secondary endpoints included time to response (time from the start of treatment to the response according to mIWGMRT-ECNM criteria); duration of response (DOR; time from first documented response to the date of the first documented disease progression/loss of response or death due to any cause, whichever occurred first); PFS (time from the start of treatment to the date of the first documented disease progression as per mIWG-MRT-ECNM criteria or death due to any cause, whichever occurred first); OS (time from the start of treatment to the date of death); and changes in mast cell burden, safety and QoL using EORTC-QLQ-C30. Additional planned secondary endpoints not reported in this manuscript included pharmacokinetics of avapritinib and morphological response based on the Pure Pathological Response criteria $^{7,26}$.

Response according to mIWG-MRT-ECNM criteria was assessed at C1D15, C2D1, C3D1, C7D1 and every six cycles thereafter, 12 weeks after documentation of CR or PR, 4 weeks after progressive disease of the AdvSM and/or AHN components and at the end of therapy if discontinued for a reason other than progressive disease or initiation of alternative cytoreductive therapy. Patient-reported outcomes (AdvSM-SAF, PGIS and EORTC-QLQ-C30) were collected at each visit through cycle 17 and at the end of therapy (if before or at cycle 17).

All patients were followed for safety (until 30 days after treatment discontinuation) and for long-term survival every 3 months. Safety assessments included determination of ECOG performance status, clinical laboratory testing, vital signs, electrocardiograms, brain imaging (magnetic resonance imaging or computerized tomography scan) and physical examinations. Treatment-emergent AEs were defined as any AE that occurred between the first dose of avapritinib through 30 days after the last dose of avapritinib, and were graded according to National Cancer Institute Common Terminology Criteria for AEs, v.5.0.

Statistical analysis. The null hypothesis ORR of $28 \%$ versus the alternative hypothesis, ORR of $50 \%$, a one-sided type I error rate of 0.025 and a sample size of approximately 63 patients in cohort 1 were determined to have $93.5 \%$ power using the exact one-sample binomial test. The null hypothesis, ORR of $28 \%$, was based on ORR as per IWG-MRT-ECNM criteria for midostaurin ${ }^{19}$. The data cutoff date for this interim analysis was 23 June 2020, and was performed when 32 patients in cohort 1 had received six cycles of treatment and at least two postbaseline bone marrow assessments or had an end-of-study assessment at any timepoint. In the interim analysis, the null hypothesis was rejected if one-sided $P<0.00625$. In the case of failure of the interim analysis, the final analysis would be tested at a one-sided alpha level of 0.02178 . Two-sided $95 \%$ confidence intervals were based on the exact binomial distribution (Clopper-Pearson method). Time-to-event outcomes (DOR, PFS and OS) were determined using the Kaplan-Meier method, and estimates were computed using Greenwood's formula. Summary statistics are presented for time to response. Maximum percentage baseline reduction in clinicopathological measures of response (bone marrow mast cells, serum tryptase, KIT D816V VAF and spleen volume) was based on patients with both baseline (last observation before the date of the first dose of avapritinib, including pre-dose assessments on this date) and at least one baseline assessment. The mean change from baseline AdvSM-SAF TSS to C11D1 was tested against the null hypothesis of $\geq 0$. If one-sided, one-sample $t$-test $P<0.025$, the null hypothesis was rejected. Summary statistics and change from baseline are presented for PGIS and QoL assessment by EORTC-QLQ-C30.

All safety analyses, QoL outcomes and secondary analyses were evaluated in the safety population, comprising all enrolled patients. All statistical analyses were conducted using SAS v.9.4 or higher.

Trial oversight and review. The trial was designed by the sponsor (Blueprint Medicines Corporation) and trial investigators. The full protocol was approved by the institutional review board (IRB) or independent ethics committee (IEC) of each participating center: St Michael's Hospital Research Ethics Board, Toronto, Canada; East of England - Cambridge South Research Ethics Committee, Nottingham, UK; University of Pennsylvania Office of Regulatory Affairs, PA, USA; Dana-Farber Cancer Institute, Office for Human Research Studies, MA, USA; University of Utah IRB, UT, USA; University of Michigan Medical School Institutional Review Board, MI, USA; MD Anderson Cancer Center, Office of Protocol Research, TX, USA; Stanford University, Research Compliance Office, CA, USA; Columbia University Medical Center IRB, NY, USA; Western IRB, WA, USA; Rush University Medical Center IRB, IL, USA; Washington University, Human Research Protocol Office, MO, USA; Roswell Park IRB, NY, USA; Region Syddanmark, Vejle, Denmark; Comité de protection des personnes Sud Est 1, Saint-Étienne, France; Ethikkommission II der Universität Heidelberg, Medizinische Fakultät, Mannheim, Germany; Medical Ethics Review Board, Groningen, the Netherlands; Comitato Etico Campania Sud - ASL; Napoli 3 Sud, Italy; Comitato Etico Area Vasta Centro, Florence, Italy; CEIC Hospital Universitari Pare Tau Ii - Oficina de Recerca, Barcelona, Spain; REK SØR/ØST, Oslo, Norway; and Independent Bioethics Committee for Scientific Research at the Medical University of Gdansk, Gdansk, Poland. The trial was conducted in accordance with the Declaration of Helsinki, International Conference on Harmonisation guidelines for Good Clinical Practice and local regulations. All patients provided written informed consent. Participants 
were not compensated, except for reimbursement of reasonable travel expenses. Each local IRB/IEC was notified based on local regulations (where required by the IRB/IEC) of all serious, unexpected adverse drug reactions involving risk to human patients. The sponsor and authors jointly collected and analyzed the data All authors had access to all data, reviewed and provided critical input to the manuscript and made the decision to submit it for publication. All authors vouch for the validity of the trial results and adherence to the protocol.

Reporting Summary. Further information on research design is available in the Nature Research Reporting Summary linked to this article.

\section{Data availability}

The anonymized derived data from this trial that underlie the results reported in this article will be made available, beginning 12 months and ending 5 years after this article's publication, to any investigators who sign a data access agreement and provide a methodologically sound proposal to medinfo@blueprintmedicines.com. The trial protocol will also be made available, as will a data fields dictionary.

\section{References}

23. Gotlib, J. et al. International Working Group-Myeloproliferative Neoplasms Research and Treatment (IWG-MRT) \& European Competence Network on mastocytosis (ECNM) consensus response criteria in advanced systemic mastocytosis. Blood 121, 2393-2401 (2013).

24. Gotlib, J. et al. Avapritinib, a potent and selective inhibitor of KIT D816V, improves symptoms of advanced systemic mastocytosis (AdvSM): analyses of patient reported outcomes (PROs) from the phase 1 (EXPLORER) study using the (AdvSM) Symptom Assessment Form (AdvSM-SAF), a new PRO questionnaire for (AdvSM). Blood 132, Suppl. 1 (2018).

25. Taylor, F. et al. Psychometric evaluation of the Advanced Systemic Mastocytosis Symptom Assessment Form (AdvSM-SAF). Leuk. Res. 108 106606 (2021)

26. Gotlib, J. et al. Pure pathologic response is associated with improved overall survival in patients with advanced systemic mastocytosis receiving avapritinib in the phase I EXPLORER study. In American Society of Hematology 62nd Annual Meeting and Exposition (Virtual, 2020).

\section{Acknowledgements}

The trial was funded by Blueprint Medicines Corporation. The Sponsor was involved in the trial design, collection, analysis and interpretation of data, as well as data checking of information provided in the manuscript. However, ultimate responsibility for opinions, conclusions and data interpretation lies with the authors. We thank the patients, their families and all investigators involved in this trial. Additional pathology support was provided by A. Rets, K. Moser and K. Karner (University of Utah/ARUP Laboratories, Salt Lake City, UT, USA). Data management support was provided by C. Langford (Stanford Cancer Institute, Stanford, CA, USA). Medical writing support, including assisting authors with the development of drafts, preparation of figures, incorporation of comments, data checking and referencing, was provided by K. Tran and C. Tomas (both of Paragon, Knutsford, UK), and editorial support, including formatting, proofreading and submission, was provided by T. Taylor (Paragon, Knutsford, UK, supported by Blueprint Medicines Corporation in accordance with Good Publication Practice guidelines (www.ismpp.org/gpp3). J.G. is supported by an internal grant from the Stanford Cancer Institute Clinical Innovation Fund. A.R. is supported by the Deutsche José-Carreras Leukämiestiftung grant (no. DJCLS 08R/2020). A.M.V. is supported in part by a grant from Association Italiana per la Ricerca sul Cancro, Mynerva project (no. 21267). E.O.H. is supported by the National Cancer Institute (National Institutes of Health (NIH) nos. 1UE5CA246744-01 and P01-CA214278-03), the National Center for Advancing Translational Sciences (NIH no. NIH-NCATS UL1TR001878) and the National Heart, Lung, and Blood Institute (NIH no. R01-HL-148014-01A1). R.M. is supported by a P30 grant (no. CA054174). P.B. is supported by the National Cancer Institute (NIH, MD Anderson Cancer Center support grant P30 no. CA016672).

\section{Author contributions}

B.G.M. was the medical monitor of the study. H.-M.L. led the generation of data analyses J.G., A.R. and D.J.D. contributed equally. All authors were involved in data interpretation and in the writing, revision and critical review of the manuscript. All authors have approved the submitted version and are accountable for their contributions and the integrity of the work.

\section{Competing interests}

J.G. is the Chair of the Study Steering Committee of the PATHFINDER trial, Chair of the Response and Adjudication Committee for the EXPLORER trial, has received research funding, served on advisory boards and received honoraria and funding to cover travel expenses from Blueprint Medicines Corporation. He has received research funding, is the co-chair of the phase 2 trial of ripretinib in AdvSM Study Steering Committee and has honoraria for these roles and serves on advisory boards for Deciphera. He also serves as Chair of the Central Response Review Committee for the phase 2 trial of bezuclastinib in AdvSM. A.R. has been a clinical advisory board/study steering committee member (PATHFINDER trial) and received honoraria and funding to cover travel expenses from Blueprint Medicines Corporation. He has received advisory board fees, speaking fees and travel support from Novartis, Deciphera, Incyte, Celgene, AOP Pharmaceuticals and AbbVie, and research support from Novartis. D.H.R. has been a clinical advisory board/study steering committee member (EXPLORER and PATHFINDER studies) for Blueprint Medicines Corporation, and was involved with educational events and advisory boards for Novartis. M.W.D. has received honorarium fees from Blueprint Medicines Corporation, Incyte, Medscape, Sangamo and Takeda; consultancy fees from Blueprint Medicines Corporation, DisperSol, Fusion Pharma, Novartis and Sangamo; and research funding from Blueprint Medicines Corporation, Incyte, Leukemia \& Lymphoma Society, Novartis, Pfizer, SPARC and Takeda. He is part of Study Steering Committees for Blueprint Medicines Corporation and Takeda and is a case author for Medscape. T.I.G. has been a clinical advisory board/study steering committee member (EXPLORER and PATHFINDER studies) and has received consulting fees from Blueprint Medicines Corporation, Celgene and Incyte. J.P. has received honorarium fees from Apellis, Blueprint Medicines Corporation, BMS, Grünenthal, MSD, Sobi and F. Hoffmann-La Roche. He has served on the speaker's bureau of Alexion, Boehringer Ingelheim, Novartis, Pfizer and Chugai. A.M.V. has participated in speakers' bureaux for AOP, BMS-Celgene, Novartis and Shire, and on advisory boards for AbbVie, BMS-Celgene, CTI BioPharma, Incyte and Novartis. U.P. received research funding from BMS, Amgen, Novartis, Curis and BerGenBio. I.A.-T. has served on advisory boards for, and received honoraria and research funding from, Blueprint Medicines Corporation and has participated in educational events for Novartis. A.M. has received honorarium fees from Takeda, Pfizer, Novo Nordisk, Behring, AbbVie, Novartis, Cilag, Janssen and Bayer. O.H. received research funding from AB Science, BMS/Celgene, Alexion, Novartis and Inatherys, consulted for $\mathrm{AB}$ Science and is a shareholder for $\mathrm{AB}$ Science. I.D. has received advisory board fees from Novartis. E.O.H. has received research support (institution) from Blueprint Medicines Corporation, Samus Therapeutics and Novartis Oncology. She serves on a data safety monitoring committee for Blueprint Medicines Corporation and is a member of the hematology exam committee for the American Board of Internal Medicine. L.K.H. has received research support (institution) from Gilead Sciences. L.S. has no disclosures. R.M has been a consultant for Novartis, Sierra Oncology and La Jolla Pharma and has received research support from Celgene, Incyte, AbbVie, Samus, Genentech, Promedior and CTI BioPharma. P.B. has received research support from Incyte, Celgene (now BMS), CTI BioPharma, Kartos, Blueprint Medicines Corporation, Constellation, Astellas, Pfizer, NS Pharma and Promedior; and honoraria from Incyte, Celgene (now BMS), CTI BioPharma, Kartos and Blueprint Medicines Corporation. K.M.P. has participated on advisory boards for CTI BioPharma, PharmaEssentia and Kura Oncology. M.L.H. has received research funding from Blueprint Medicines Corporation, BMS, CTI BioPharma, Deciphera, Incyte, Novartis and Sierra Oncology; and consulting fees from AbbVie, CTI BioPharma, Novartis, Blueprint Medicines Corporation and Partner Therapeutics. S.T.O. has been a consultant for Gilead Sciences, Novartis, Kartos Therapeutics, CTI BioPharma, Celgene/ Bristol Myers Squibb, Disc Medicine, Blueprint Medicines Corporation, PharmaEssentia, Constellation and Incyte. J.S., H.-M.L. and B.G.M. are employees and/or equity holders of Blueprint Medicines Corporation. D.J.D. has been a clinical advisory board/study steering committee member (EXPLORER and PATHFINDER studies) for Blueprint Medicines Corporation. He has served as a consultant for Amgen, Agios, Autolus, Forty-Seven, Incyte Corporation, Jazz, Novartis, Pfizer, Shire and Takeda; and has received research funding from AbbVie, GlycoMimetics and Novartis.

\section{Additional information}

Extended data is available for this paper at https://doi.org/10.1038/s41591-021-01539-8. Supplementary information The online version contains supplementary material available at https://doi.org/10.1038/s41591-021-01539-8.

Correspondence and requests for materials should be addressed to Jason Gotlib. Peer review information Nature Medicine thanks Jongphil Kim, Michael Mauro and Guillermo Garcia-Manero for their contribution to the peer review of this work. Primary Handling Editor: Saheli Sadanand, in collaboration with the Nature Medicine team.

Reprints and permissions information is available at www.nature.com/reprints. 


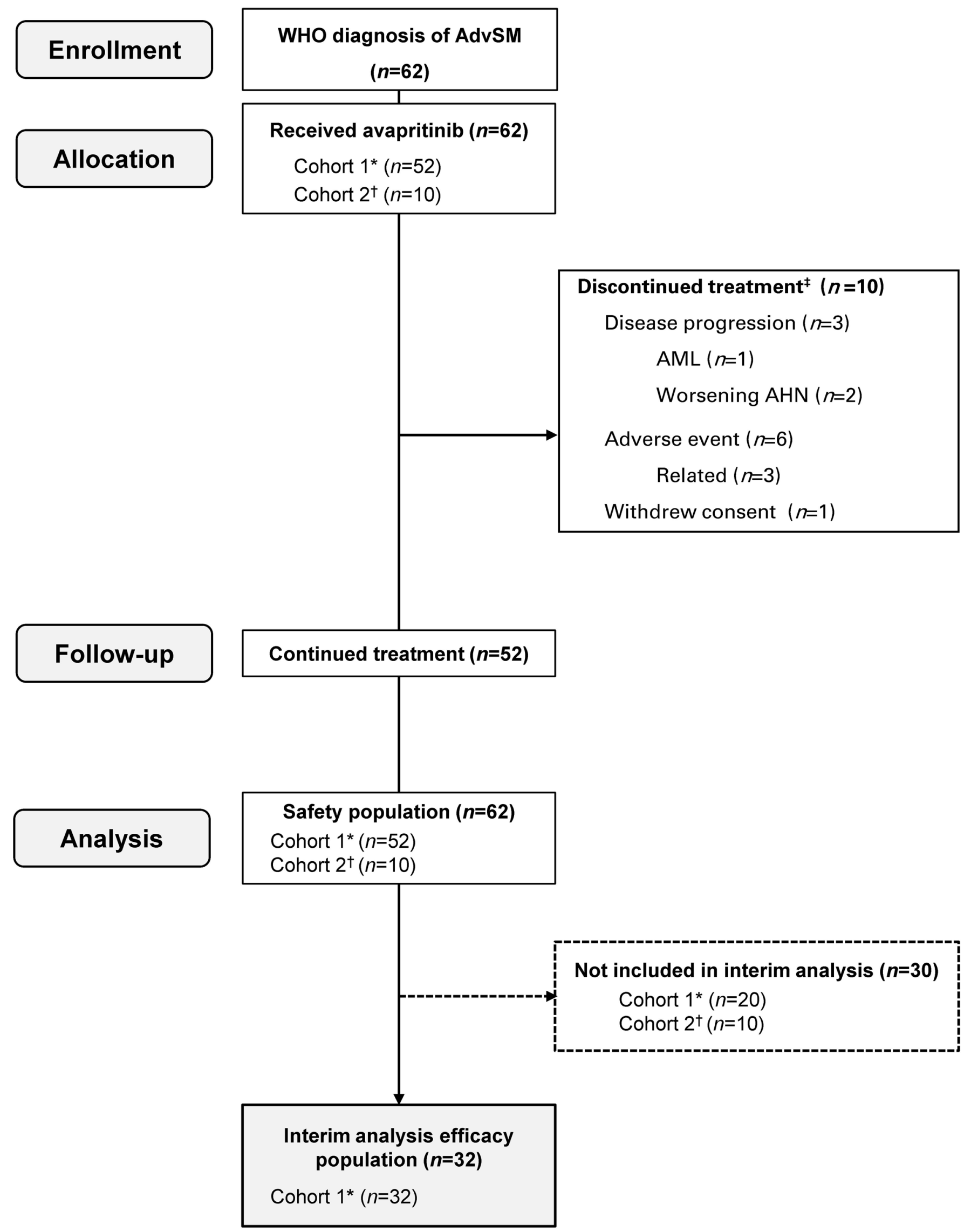


Extended Data Fig. 1 | Patient Disposition. *Cohort 1: Patients with AdvSM and $\geq 1$ mIWG-MRT-ECNM criteria for evaluable disease (have severe and quantifiable organ damage (an evaluable C-finding) or have MCL (regardless of C-findings)) as confirmed by the SSC. Twenty patients in Cohort 1 were not included in the interim analysis efficacy population due to lack of sufficient follow-up. Patients with AdvSM who were not considered eligible for an adjudicated response and were confirmed centrally to have ASM or SM-AHN, but were lacking an evaluable C-finding as determined by the SSC. No patient discontinued from treatment due to lost to follow-up, protocol deviation, pregnancy, investigator decision, non-compliance, sponsor decision, or other reason. AdvSM, advanced systemic mastocytosis; AHN, associated hematologic neoplasm; AML, acute myeloid leukemia; ASM, aggressive systemic mastocytosis; MCL, mast cell leukemia; mIWG-MRT-ECNM, modified International Working Group-Myeloproliferative Neoplasms Research and Treatment and European Competence Network on Mastocytosis; SM-AHN, systemic mastocytosis with an associated hematologic neoplasm; SSC, study steering committee; WHO, World Health Organization. 
Prior Treatment

\section{Patients Receiving Prior}

Anti-Neoplastic Therapy, $n(\%)$
Any

Midostaurin

Cladribine

Imatinib

Hydroxycarbamide

Azacitidine

Interferon alfa

Dasatinib

Decitabine

Investigational antineoplastic drugs

Hematopoietic stem cell transplantation

Thalidomide
$23(72)$

$17(53)$

$4(13)$

$4(13)$

$3(9)$

$2(6)$

$2(6)$

$1(3)$

$1(3)$

$1(3)$

$1(3)$

$1(3)$

Extended Data Fig. 2 | Prior Anti-Neoplastic Therapies (Interim Analysis Efficacy Population, $\boldsymbol{n}=\mathbf{3 2}$ ). Table shows details of prior therapies received by patients that ended on or before the first dose date of avapritinib. 


\begin{tabular}{|c|c|c|c|}
\hline & $\begin{array}{l}\text { Safety Population } \\
\qquad(n=62)\end{array}$ & $\begin{array}{c}\text { Efficacy } \\
\text { Population } \\
(n=32)\end{array}$ & $\begin{array}{c}\text { included in Interim } \\
\text { Analysis } \\
(n=30)\end{array}$ \\
\hline Median age, years (range) & $69(31-88)$ & $68(37-85)$ & $70(31-88)$ \\
\hline Male / female, $n(\%)$ & $34(55) / 28(45)$ & $18(56) / 14(44)$ & $16(53) / 14(47)$ \\
\hline \multicolumn{4}{|l|}{ ECOG performance status, $n(\%)$} \\
\hline $0-1$ & $43(69)$ & $21(66)$ & $22(73)$ \\
\hline $2-3$ & $19(31)$ & $11(34)$ & $8(27)$ \\
\hline \multicolumn{4}{|l|}{ AdvSM subtype per central assessment, $n(\%)$} \\
\hline ASM & $9(15)$ & $2(6)$ & $7(23)$ \\
\hline SM-AHN & $43(69)$ & $26(81)$ & $17(57)$ \\
\hline $\mathrm{MCL}$ & $10(16)$ & $4(13)$ & $6(20)$ \\
\hline \multicolumn{4}{|l|}{$\begin{array}{l}\text { KIT D816V mutation status in peripheral blood } \\
\text { by central ddPCR, } n(\%)\end{array}$} \\
\hline Positive & $59(95)$ & $30(94)$ & $29(97)$ \\
\hline Negative & $3(5)$ & $2(6)$ & $1(3)$ \\
\hline $\begin{array}{l}\text { KIT D816V variant allele fraction in blood, } \\
\text { median percent (range) }\end{array}$ & $18(0-47)$ & $15(0-45)$ & $19(0-47)$ \\
\hline \multicolumn{4}{|l|}{$\begin{array}{l}\text { SRSF2/ASXL1/RUNX1 mutation per central } \\
\text { assay, } n(\%)\end{array}$} \\
\hline Positive & $26(42)$ & $17(53)$ & $9(30)$ \\
\hline Negative & $36(58)$ & $15(47)$ & $21(70)$ \\
\hline \multicolumn{4}{|l|}{ Prior anti-neoplastic therapy, $n(\%)$} \\
\hline Any & $42(68)$ & $23(72)$ & $19(63)$ \\
\hline Midostaurin & $34(55)$ & $17(53)$ & $17(57)$ \\
\hline Cladribine & $8(13)$ & $4(13)$ & $4(13)$ \\
\hline Imatinib & $5(8)$ & $4(13)$ & $1(3)$ \\
\hline Interferon & $6(10)$ & $2(6)$ & $4(13)$ \\
\hline $\begin{array}{l}\text { Bone marrow biopsy mast cell burden, } \\
\text { median percent (range) }\end{array}$ & $45(1-95)$ & $50(10-95)$ & $35(1-90)$ \\
\hline Serum tryptase level, median ng/mL (range) & $283(24-1600)$ & $293(24-1600)$ & $276(31-1208)$ \\
\hline Spleen volume, median $\mathrm{mL}$ (range) & $748(44-2601)$ & $939(150-2270)$ & $671(44-2601)$ \\
\hline
\end{tabular}

Extended Data Fig. 3 | Baseline Characteristics. Baseline characteristics including for patients not included in the interim analysis ( $n=30$ ). AdvSM, advanced systemic mastocytosis; ASM, aggressive systemic mastocytosis; ddPCR, droplet digital polymerase chain reaction; ECOG, Eastern Cooperative Oncology Group; MCL, mast cell leukemia; mIWG-MRT-ECNM, modified International Working Group-Myeloproliferative Neoplasms Research and Treatment and European Competence Network on Mastocytosis; SM-AHN, systemic mastocytosis with associated hematologic neoplasm. 
Presence of Evaluable C-Findings per mIWG-MRT-ECNM criteria*
Patients with

C-Finding at

Baseline, $n$ (\%)
Patients with Clinical Improvement in C-finding, $n(\%)^{\dagger}$
Median Time to Clinical Improvement, months

\begin{tabular}{lccc}
\hline Ascites & $7(22)$ & $4(57)$ & 3.3 \\
\hline Pleural effusions & $6(19)$ & $5(83)$ & 3.8 \\
\hline Liver function abnormalities & & & - \\
Direct Bilirubin $>1.5 \times$ ULN & $2(6)$ & 0 & - \\
ALT >3.0 $\times$ ULN & 0 & - & - \\
AST $>3.0 \times$ ULN & 0 & - & 3.8 \\
ALP >2.5 $\times$ ULN & $13(41)$ & $5(38)$ & 0.5 \\
\hline Hypoalbuminemia & $3(9)$ & $1(33)$ & 1.9 \\
\hline Splenomegaly & $14(44)$ & $11(79)$ & 5.6 \\
\hline Neutropenia & $2(6)$ & $1(50)$ & \\
\hline Anemia & & & 3.8 \\
Transfusion-independent & $13(41)$ & $5(38)$ & 5.8 \\
\hline Transfusion-dependent & $3(9)$ & $1(33)$ & 4.2 \\
\hline Thrombocytopenia & & $1(17)$ & - \\
Transfusion-independent & $6(19)$ & 0 & \\
Transfusion-dependent & $2(6)$ & & \\
\hline
\end{tabular}

Extended Data Fig. 4 | Response by mIWG-MRT-ECNM C-Findings (Interim Analysis Efficacy Population, $\mathbf{n}=32$ ). ${ }^{\star}$ Definitions of C-findings per mIWG-MRT-ECNM and criteria for measuring clinical improvement are presented in Supplementary Table 1 and Supplementary Table 2. ${ }^{\dagger}$ Percentages are based on the number of patients with C-finding at baseline. ALP, alkaline phosphatase; ALT, alanine aminotransferase; AST, aspartate aminotransferase; mIWG-MRT-ECNM, modified International Working Group-Myeloproliferative Neoplasms Research and Treatment and European Competence Network on Mastocytosis; ORR, overall response rate; ULN, upper limit of normal. 


\section{NATURE MEDICINE}

a

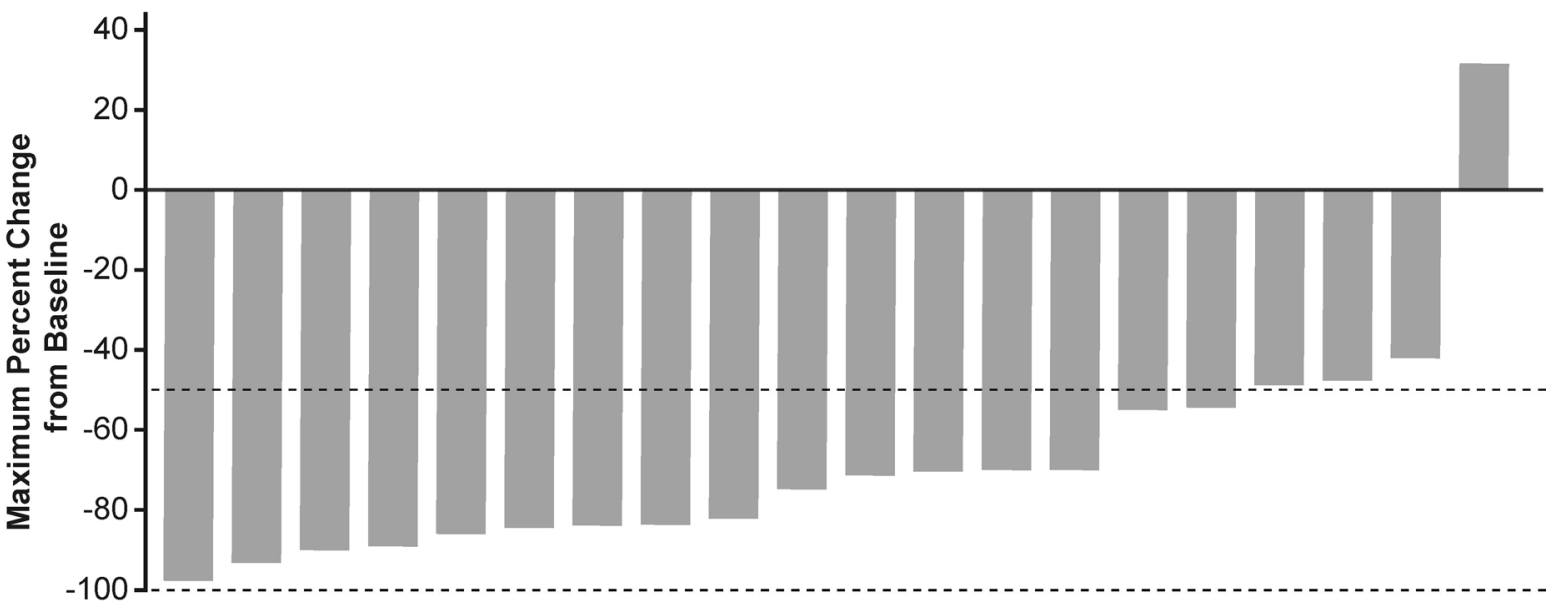

b

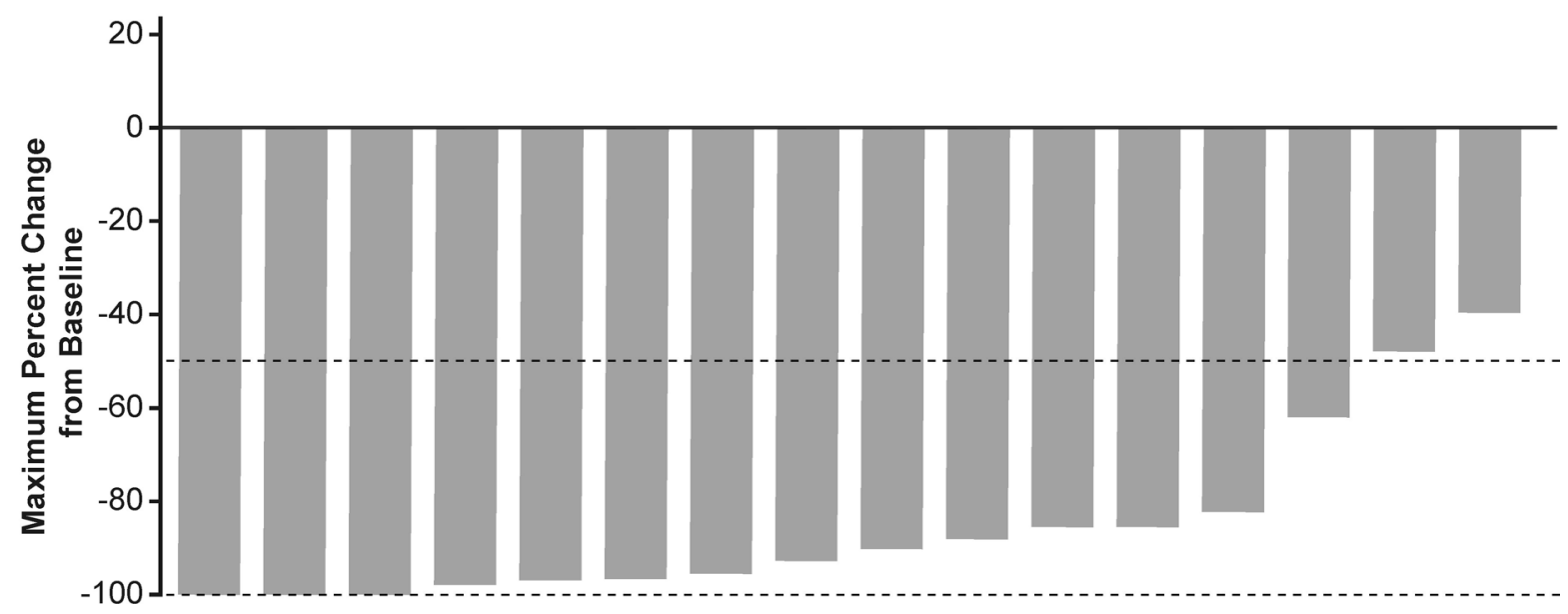

Extended Data Fig. 5 | Change from Baseline in Clinicopathological Measures of Disease Burden. a, Peripheral blood absolute monocyte count in patients with SM-CMML. b, Peripheral blood absolute eosinophil count in patients with baseline eosinophilia. SM-CMML, systemic mastocytosis with chronic myelomonocytic leukemia. 
All Doses $(n=62)$

\begin{tabular}{lcc} 
& Any Grade & Grade $\geq 3$ \\
\cline { 2 - 3 } Any AE leading to dose interruption, $n(\%)$ & $34(55)$ & $31(50)$ \\
AEs leading to dose interruption in $\geq 5 \%, n(\%)$ & $13(21)$ & $13(21)$ \\
Neutropenia* & $10(16)$ & $9(15)$ \\
Thrombocytopenia* & $3(5)$ & $3(5)$ \\
Leukopenia* $^{*}$ & $42(68)$ & $29(47)$ \\
\hline Any AE leading to dose reduction, $n(\%)$ & & $12(19)$ \\
AEs leading to dose reduction in $\geq 5 \%, n(\%)$ & $12(19)$ & $8(13)$ \\
Neutropenia* & $11(18)$ & $1(2)$ \\
Thrombocytopenia* & $6(10)$ & $1(2)$ \\
Peripheral edema & $4(6)$ & $4(6)$ \\
Periorbital edema* & $6(10)$ & 0 \\
\hline Any AE leading to treatment discontinuation, $n(\%)$ & $1(2)$ & 0 \\
Decreased weight & $1(2)$ & $1(2)$ \\
Hemorrhagic diathesis ${ }^{\dagger}$ & $1(2)$ & $1(2)$ \\
Hemorrhagic shock & $1(2)$ & $1(2)$ \\
Necrotizing fasciitis & $1(2)$ & $1(2)$ \\
Subdural hematoma & $1(2)$ & \\
Thrombocytopenia* & & \\
\hline
\end{tabular}

Extended Data Fig. 6 | Adverse Events Leading to Dose Modifications. * Pooled terms. ${ }^{\dagger}$ Patient reported to have increased bleeding propensity with subcutaneous hematoma. $\mathrm{AE}$, adverse event. 


\section{Reporting Summary}

Nature Research wishes to improve the reproducibility of the work that we publish. This form provides structure for consistency and transparency in reporting. For further information on Nature Research policies, see our Editorial Policies and the Editorial Policy Checklist.

\section{Statistics}

For all statistical analyses, confirm that the following items are present in the figure legend, table legend, main text, or Methods section.

$\mathrm{n} / \mathrm{a}$ Confirmed

$\bigotimes$ The exact sample size $(n)$ for each experimental group/condition, given as a discrete number and unit of measurement

Х $\square$ A statement on whether measurements were taken from distinct samples or whether the same sample was measured repeatedly

The statistical test(s) used AND whether they are one- or two-sided

Only common tests should be described solely by name; describe more complex techniques in the Methods section.

Х A description of all covariates tested

Х $\square$ A description of any assumptions or corrections, such as tests of normality and adjustment for multiple comparisons

$\square$ A full description of the statistical parameters including central tendency (e.g. means) or other basic estimates (e.g. regression coefficient) AND variation (e.g. standard deviation) or associated estimates of uncertainty (e.g. confidence intervals)

For null hypothesis testing, the test statistic (e.g. $F, t, r$ ) with confidence intervals, effect sizes, degrees of freedom and $P$ value noted Give $P$ values as exact values whenever suitable.

Х $\square$ For Bayesian analysis, information on the choice of priors and Markov chain Monte Carlo settings

$\bigotimes \square$ For hierarchical and complex designs, identification of the appropriate level for tests and full reporting of outcomes

$\triangle \square$ Estimates of effect sizes (e.g. Cohen's $d$, Pearson's $r$ ), indicating how they were calculated

Our web collection on statistics for biologists contains articles on many of the points above.

\section{Software and code}

Policy information about availability of computer code

Data collection No software used

Data analysis SAS version 9.4

For manuscripts utilizing custom algorithms or software that are central to the research but not yet described in published literature, software must be made available to editors and

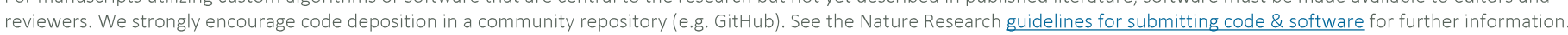

\section{Data}

Policy information about availability of data

All manuscripts must include a data availability statement. This statement should provide the following information, where applicable:

- Accession codes, unique identifiers, or web links for publicly available datasets

- A list of figures that have associated raw data

- A description of any restrictions on data availability

The anonymized derived data from this trial that underlie the results reported in this article will be made available, beginning 12 months and ending 5 years after this article's publication, to any investigators who sign a data access agreement and provide a methodologically sound proposal to medinfo@blueprintmedicines.com. The trial protocol will also be made available, as will a data fields dictionary. 
Please select the one below that is the best fit for your research. If you are not sure, read the appropriate sections before making your selection.

\ Life sciences

Behavioural \& social sciences

Ecological, evolutionary \& environmental sciences

For a reference copy of the document with all sections, see nature.com/documents/nr-reporting-summary-flat.pdf

\section{Life sciences study design}

All studies must disclose on these points even when the disclosure is negative.

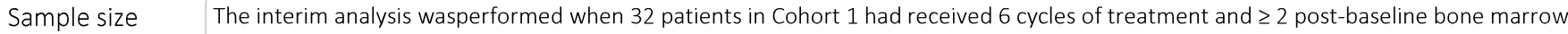
assessments or had an end-of-study assessment at any timepoint. The null hypothesis ORR of $28 \%$ was based on the ORR per IWG-MRT-ECNM criteria for midostaurin. 9 In the interim analysis, the null hypothesis rejected if the 1 -sided $P<.00625$. In the case of failure of the interim analysis, a sample size of approximately 63 patients in Cohort 1 at final analysis was determined to have $93.5 \%$ power at the 1-sided significance level of 0.025 for the null hypothesis ORR of $28 \%$ versus the alternative hypothesis ORR of $50 \%$.

Data exclusions No data exclusions

Replication N/A. This was an interim analysis of a non-randomized Phase 2 clinical study in patients with advanced systemic mastocytosis.

Randomization Single-arm study-no randomization

Blinding Single-arm study - no blinding

\section{Reporting for specific materials, systems and methods}

We require information from authors about some types of materials, experimental systems and methods used in many studies. Here, indicate whether each material, system or method listed is relevant to your study. If you are not sure if a list item applies to your research, read the appropriate section before selecting a response.

\begin{tabular}{l|l} 
Materials \& experimental syste \\
\hline n/a & Involved in the study \\
\hline & $\square$ Antibodies \\
$\square$ & $\square$ Eukaryotic cell lines \\
$\square$ & $\square$ Animals and other organisms \\
$\square$ & $\square$ Clinical data \\
$\square$ & $\square$ Dual use research of concern
\end{tabular}

\begin{tabular}{l|l}
\multicolumn{2}{l}{ Methods } \\
\hline n/a & Involved in the study \\
$\square$ & $\square$ ChIP-seq \\
$\square$ & $\square$ Flow cytometry \\
$\square$ & $\square$ MRI-based neuroimaging
\end{tabular}

Human research participants

Policy information about studies involving human research participants

Population characteristics

Recruitment

Ethics oversight
N/A - no covariate analyses conducted. The patient demographics are described in full in the manuscript (Table 1). Of all 62 enrolled patients, 28 (45\%) were female and 34 (55\%) were male. The median participant age was 69 years (range $31-88$ years)

Patients were recruited by participating investigators. The Investigator at each center ensured that the patients were given full and adequate oral and written information about the nature, purpose, possible risk, and benefit of the study. Patients were also notified that they were free to discontinue from the study at any time. Patients were given the opportunity to ask questions and allowed time to consider the information provided. Inclusion and exclusion criteria are defined in full in tables in the manuscript Supplementary Information. All patients provided written informed consent. Participants were not compensated, except for the reimbursement of reasonable travel expenses.

Patients were enrolled across 18 study centers in North America and Europe. Due to the geographical distribution of the study centers, participants may not represent the global general population. No other bias emerging from recruitment is expected.

The full protocol was approved by the institutional review board or independent ethics committee of each participating center, and written informed consent was obtained from all participants. 
Policy information about clinical studies

All manuscripts should comply with the ICMJE guidelines for publication of clinical research and a completed CONSORT checklist must be included with all submissions.

Clinical trial registration

Study protocol

Data collection

Outcomes
ClinicalTrials.gov: PATHFINDER study (NCT03580655)

Requests for the study protocol should be directed to the Sponsor via medinfo@blueprintmedicines.com

Patients recruited and data collected at hospitals and medical centers between November 2018 and June 2020 (data cut-off): 28 patients were enrolled at 10 sites in North America, and 34 patients at 8 sites in Europe.

Primary endpoint: overall response rate as assessed by mIWG-MRT-ECNM criteria. Key secondary endpoint was change in total symptom score per AdvSM-SAF. Other secondary endpoints: duration of response, changes in mast cell burden (number of bone marrow mast cells, serum tryptase levels, KIT D816V variant allele fraction, and spleen volume), and safety per adverse events

(National Cancer Institute Common Terminology Criteria for AEs, version 5.0), changes in vital signs, electrocardiograms, and laboratory testing. 